\title{
Zukunft der Kinder- und Jugendpsychiatrie in Österreich
}

\author{
Leonhard Thun-Hohenstein (D) - Rainer FliedI · Kathrin Sevecke · Andreas Karwautz
}

Eingegangen: 15. Juni 2017 / Angenommen: 22. Juli 2017 / Online publiziert: 30. August 2017

(C) Der/die Autor(en) 2017. Dieser Artikel ist eine Open-Access-Publikation.

Zusammenfassung Die Kinder- und Jugendpsychiatrie ist nun als eigenes Sonderfach 10 Jahre alt. Das ist eine Errungenschaft, aber vielmehr noch der Auftrag die Versorgung der Kinder und Jugendlichen in Österreich auf ein jeweils besseres Niveau zu entwickeln. Vor einem politischen Hintergrund, der von Finanzknappheit und Machbarkeit dominiert ist, gilt es in kraft- und zeitaufwändiger Beziehungsarbeit Kindern und Jugendlichen zu helfen ihre Entwicklungsaufgaben zu meistern. Dazu sind vonseiten der Politik auf allen Versorgungsebenen die nötigen Mindestressourcen zur Verfügung zu stellen und in den komplementären Bereichen (Kinder- und Jugendhilfe, Sozialbereich, Justiz etc.) die nötigen komplementären und kooperativen Versorgungsstrukturen vorzuhalten. Die Ausbildungskapazität muss weiter gesteigert und die Forschungsmaßnahmen verstärkt werden. Dies alles auf Basis der Menschen- resp. Kinderrechtskonvention, um eine humane und kind- wie jugendgerechte Kinder- und Jugendpsychiatrie zu gewährleisten.

\section{A. Karwautz}

Universitätsklinik für Kinder- und Jugendpsychiatrie,

Medizinische Universität Wien, Wien, Österreich

\section{K. Sevecke}

Universitätsklinik für Psychiatrie, Psychotherapie und Psychosomatik des Kindes und Jugendalters, Universitätskliniken Innsbruck, Innsbruck, Österreich

\section{R. Fliedl}

Klinische Abteilung für Kinder- und

Jugendneuropsychiatrie, Karl Landsteiner Privatuniversität, Krankenhaus Mödling, Mödling, Österreich

\section{Thun-Hohenstein ( $\square)$}

Universitätsklinik für Kinder- und Jugendpsychiatrie,

Universitätsklinikum SALK/PMU, Ignaz

Harrerstrasse 49, 5020 Salzburg, Österreich

L.Thun-Hohenstein@salk.at
Schlüsselwörter Zukunft · Entwicklung; Kinder- und Jugendpsychiatrie $\cdot$ Kinderrechte

\section{Future development of child and adolescent psychiatry in Austria}

Summary Anniversaries are turningpoints for reflexions of the past and planing for the future. In conclusion of all papers in this actual issue one can say tha CAP has reached a respected place in the medical landscape. Also internationally, the work of Austrian child and adolescent psychiatrist is recognised and respected. CAP is cooperating as an respected discipline with adjacent specialties as pediatrics and psychiatry as well as with external partners as Kinder- und Jugendhilfe (social child welfare).

Anyway, the actual situation shows several deficits concerning patient care on all levels of the health system as well as in education and training of sufficient numbers of child and adolescent psychiatrists.

Keywords Future - Development - Child and adolescent psychiatry $\cdot$ Children rights

\section{Einleitung}

Jubiläen sind neben dem Rückblick auch immer Anlass für Überlegungen, wie die Zukunft des Jubilars weitergehen soll. In Zusammenfassung der Artikel dieses Heftes kann man feststellen, dass die Kinder- und Jugendpsychiatrie in der medizinischen Landschaft Österreichs ihren Platz gefunden hat und sich auch international an verschiedenen Fachgesellschaften aktiv beteiligt [18]. Sie arbeitet als anerkannte medizinische Fachdisziplin, ist vernetzt und kooperiert mit den Nachbarfächern und den sie umgebenden Strukturen, wie Kinder- und Jugendhilfe, sie forscht, sie lehrt und ist politisch sehr aktiv, wie dies dem 
Charakter dieses Faches implizit zugehörig ist [9]. Dennoch zeigt die Analyse der aktuellen Situation, dass es viele Baustellen gibt und vieles noch nicht zur Zufriedenheit gelungen ist. Daher seien einige Punkte für die zukünftige Entwicklung des Fachgebietes diskutiert.

\section{Gesellschaftlicher Hintergrund}

Um ein Zukunftsszenario zu entwerfen, müssen wir einen kurzen Blick in unsere Zeit werfen und in die Lebenswelten unserer Kinder und Jugendlichen. Die klassische Familie wird zunehmend durch andere Modelle ersetzt (Stichwort: Patchwork-Familien), die meisten Kinder sind früh in Kinderversorgungseinrichtungen integriert und beide Eltern berufstätig. Leistungserbringung ist ein vorrangiges Thema, die Überwachung der Kinder ist heute wesentlich stärker als vor 20 oder 40 Jahren - Stichwort: Helikoptereltern. Die Natur als quasi normale Umwelt tritt immer weiter in den Hintergrund und findet hauptsächlich über die Nachhaltigkeitsdiskussion und die Bedrohung durch den Klimawandel Eingang in die kindlichen Welten. Ein wesentlicher Wandel hat im Bereich der technologischen Ausstattung der heutigen Kinder und Jugendlichen stattgefunden, in Deutschland und Österreich haben mehr als $95 \%$ der Kinder und Jugendlichen Smartphones und Internetzugang, sie nutzen diese Geräte täglich mindestens einmal und verbringen im Durchschnitt $200 \mathrm{~min}$ an diesen Geräten [10]. Das Bedrohungsgefühl [21] hat sich durch die politische Nähe zu großen Konflikten (Syrienkrise etc.), durch die Flüchtlingswanderungen und durch teilweise nur vermeintliche Folgen wie Arbeitslosigkeit und sinkende Einkommen zugenommen.

Die Häufigkeit kinder- und jugendpsychiatrischer Erkrankungen ist an sich durch die letzten Jahrzehnte mit ca. $20 \%$ relativ hoch konstant geblieben, jüngst publizierte Daten aus Österreich weisen aber auf eine weitere Zunahme hin [29]. Es gibt auch innerhalb der Diagnosen Verschiebungen wie die $\mathrm{Zu}$ nahme bestimmter klinischer Bilder (Zunahme von Essstörungen, selbstverletzendes Verhalten), durch das zunehmend frühere Erkrankungsalter bei sogenannt „Erwachsenen-typischen Erkrankungen“ und das Hinzukommen neuer Krankheitsbilder (z. B. Genderdysphorie, Internetsucht, Cybermobbing, Schlafstörungen i. Zus. mit Nutzung elektronischer Medien) und durch die forschungsbedingte Veränderung der medizinischen Nomenklaturen (z. B. DSM-V [1]).

Die Kinder- und Jugendpsychiatrie ist mit ihrer unmittelbaren Nähe zu Kindern und Jugendlichen ein „Seismograph“ für gesellschaftliche Veränderungen [9] und hat mit den Folgen gesellschaftlicher Veränderungen sehr schnell direkt zu tun. Aus dieser Nähe zu einem - für die Zukunft - wichtigen Teil der Gesellschaft entsteht eine wichtige Rolle der Kinderund Jugendpsychiatrie im sozialpolitischen Gefüge, weswegen die Kinder- und JugendpsychiaterInnen auch in vielen gesellschaftpolitischen Bereichen von sich aus aktiv sind [26]. Wünschenswert wäre für die Zukunft, dass die Vertreter_innen dieses Faches von der Politik, aber auch von den Amtsstrukturen des Landes vermehrt wahrgenommen und in wesentliche - die Kinder und Jugendlichen betreffende Entscheidungsprozesse integriert werden.

\section{Versorgung}

Zum derzeitigen Standpunkt befindet sich sowohl die ambulante als auch die tagesklinische und stationäre Versorgung in etwa der Hälfte des, durch das Ministerium definierten Versorgungsniveaus [17]. Daher bedarf es hier größter Anstrengungen, diese Defizite zu verändern. Das ist abhängig von den Vorgaben der Zahler (Bundesländer) und dem politischen Willen, die Vorgaben des Ministeriums [13] umzusetzen.

Unabhängig von der stationären Versorgung sind dringend neue Konzepte anzudenken bzw. zum Teil schon umsetzbar: Der in der Kinder- und Jugendpsychiatrie immanente Ansatz der „person-centered-care"[31] muss als Grundprinzip medizinischen Handelns weiterentwickelt werden und damit neueren Betreuungskonzepten wie Home Treatment [3] eine neue Form der Nachbehandlung zu Hause nach stationärem Aufenthalt und Integrativen Konzepten [22] Raum und Finanzierung bringen. Es geht dabei um die Sicherstellung des Behandlungsangebotes in erforderlicher Qualität, eine Flexibilisierung der Behandlungsmöglichkeit, die Förderung des Prinzips „ambulant vor stationär“, um die intensive Vernetzung der Angebote und ein damit verknüpftes hochqualitatives Case Management und eine Reduktion des berühmten Drehtüreffektes [6]. Gegenargumente finden sich häufig in den heute dominierenden Strömungen, die die Medizin als Geschäftsmodell sehen und daher mehrheitlich ökonomische Maßstäbe anlegen und Effizienz, Berechenbarkeit, Vorhersehbarkeit und Kontrolle als qualitätssteuernde Parameter heranziehen [16] - ein Prozess, der als McDonaldisierung in die Diskussion Einzug gehalten hat. Vielmehr bräuchte es ein volkswirtschaftliches Herangehen, da eine bessere Behandlung, wenn möglich früher im Verlauf, zu einer besseren Prognose führt und die Behandelten zu vollwertigen Steuerzahlern befähigt, was wiederum dem Staat zu Gute kommt, zumindest in der Reduktion der Sozial- oder Justizleistungen. Gerade das Home Treatment konnte zeigen, dass sich durch diese neue Herangehensweise Spitalsaufenthalte verkürzen lassen und insgesamt bei zumindest gleichem Erfolg die Kosten pro Patient deutlich sinken [3].

Der große Spardruck im Gesundheitssystem, die Optimierungsversuche der Prozesse und die gleichzeitige Inanspruchnahme des medizinischen Personals für Dokumentationszwecke gefährdet gerade Fächer mit hohem Beziehungsanteil als Teil des fachspezifischen Know-hows. Auch das „exzessive Machbarkeits- 
denken“ [20] bringt Fächer wie das unsere in eine schwierige Situation, rasch mach- und heilbar ist in unserem Fach gar nichts - jede Intervention bedarf des Know-hows und vor allem Zeit und fachlicher Reflexion (Supervision etc.) - und die kostet wieder Zeit. Konkrete Versorgungsdefizite finden sich im medizinischen Bereich vor allem bei Spezialangeboten für Kinder und Jugendliche mit bestimmten Erkrankungen: Autismus, Essstörungen, chronisch kranken und armen Kindern. Ein Spezialthema bildet die Rehabilitation für Kinder und Jugendliche, das zur Zeit durch die öffentliche Hand neu organisiert wurde. In diesem Prozess sind zu unserem großen Bedauern die Anforderung unseres Faches komplett ignoriert worden und - nicht nachvollziehbar - sogar das Diagnosespektrum der Kinder- und Jugendpsychiatrie der Pädiatrie zugeordnet und damit aus dem Verantwortungsbereich unseres Faches entzogen.

\section{Menschenrechtskonvention}

Die Geschichte der Kinder- und Jugendpsychiatrie ist von einigen gröbsten Menschenrechtsverletzungen geprägt, auch hier in Österreich. In den letzten Jahren konnte gezeigt werden, dass die Kinderpsychiatrie diesen historischen Auftrag, derlei Vorkommnisse präventiv anzugehen, verstanden hat und ihn auch entsprechend versucht umzusetzen, wie der Artikel von Ernst Berger [2] zeigt. Dennoch bedarf es tagtäglich größter Bemühungen, die Menschenrechtskonvention [14], aber auch die Konvention der Rechte behinderter Menschen [15] in die Tat umzusetzen. Eines der Anliegen der Österreichischen Gesellschaft für Kinder- und Jugendpsychiatrie ist es in diesem Zusammenhang ein neues Unterbringungsgesetz zu erreichen, in dem diesen Menschenrechtsgedanken und gleichzeitig der qualitätsvollen Versorgung der Kinder und Jugendlichen Rechnung getragen werden soll. Dies auch vor dem Hintergrund, dass zum Teil immer noch Kinder und Jugendliche entgegen den Kinderrechten auf Erwachsenenabteilungen geschlossen untergebracht werden müssen, da die entsprechenden Einrichtungen der Kinder- und Jugendpsychiatrie fehlen [19]. Eine wesentliche Voraussetzung zur Vermeidung von Menschenrechtsverletzungen sind persönliche und Team-bezogene Supervision, regelmäßige Fallarbeit und eine sehr klare, auf ethischen und psychotherapeutischen Grundsätzen fundierte Organisationsstruktur [12, 24].

\section{Ausbildung}

Durch die „Fachärzteausbildung Neu” Fachärzteausbildung Neu [4] und die damit verbundene Integration der Ausbildung in psychotherapeutischer Medizin und die Facharztausbildung hat sich eine ganz neue Situation ergeben. Zum einen sind die Abteilungsleiter verpflichtet, bestimmte Ausbildungsinhalte der psychotherapeutischen Medizin nun in die Facharzt- ausbildung $\mathrm{zu}$ integrieren und dafür Strukturen $\mathrm{zu}$ schaffen. Einerseits sind dies Balintgruppen, regelmäßige theoretische Fortbildungen, Psychotherapie unter Supervision u. ä. und andrerseits müssen die Freistellungen gewährleistet werden, die die Teilnahme an den Theorieblöcken des von der Fachgesellschaft organisierten Curriculums ermöglichen.

Ein weiterer neuer Punkt ist die Weiterbildung kinder- und jugendpsychiatrischer Qualifikation in KJPForensik. Die forensisch tätigen Kinder- und Jugendpsychiater sind Fachärzte für Kinder- und Jugendpsychiatrie und -psychotherapie, die sich in ihren Tätigkeiten auf das Erstatten von jugendstrafrechtlichen sowie zivilrechtlichen Gutachten und die Durchführung von - durch die Behörden angeordneten - forensischen Behandlungen Minderjähriger konzentrieren. Sie müssen daher in diesem Fachgebiet über ein spezialisiertes und spezifisches Fachwissen verfügen. In Österreich hat eine spezifische zweijährige kinder- und jugendforensische Weiterbildung, die durch die Österreichische Ärztekammer als ÖÄK-Diplomfortbildung anerkannt ist, erstmalig im Februar 2016 gestartet. Sie findet im Wechsel an der Univ.-Klinik für Psychiatrie, Psychotherapie und Psychosomatik des Kindes- und Jugendalters Innsbruck und der Univ.Klinik für Kinder- und Jugendpsychiatrie der Paracelsus Medizinischen Privatuniversität Salzburg statt. Im praktischen Teil der Weiterbildung muss der Kandidat entweder eine spezifische Anzahl von Gutachten bearbeiten oder eine bestimmte Anzahl von deliktorientierten, störungsspezifischen Behandlungen oder aber eine Kombination aus beidem absolvieren. Insgesamt gliedert sich die Weiterbildung in $84 \mathrm{~h}$ Theorie und $24 \mathrm{~h}$ Praxis (Inhalte siehe unten) und beschließt mit einer mündlichen Prüfung.

\section{Qualitätssicherung}

In einem Fach wie der Kinder- und Jugendpsychiatrie vor dem Hintergrund seiner Geschichte und den großen Schwierigkeiten, wie die Off-Label Verwendung von Psychopharmaka oder unklare Dosierungsschemata, ist es eine zwingende Konsequenz die eigene Tätigkeit kritisch auf ihre Auswirkungen zu untersuchen. Es ist daher nötig, qualitätssichernde Maßnahmen zu entwickeln - wie dies im Moment durch die Qualitätssicherungskommission der ÖGKJP begonnen hat - und andererseits deren Umsetzung und Outcome zu überprüfen. Bei der Überprüfung ist wiederum unter Berücksichtigung der Menschenrechtskonvention dringend anzuraten neben klassischen Plan-Do-Check-Act-(PDCA)-Modellen [30] so genannte patient-related-outcome-measures (PROM, [5]) einzuführen und die vorhandenen Standards und Kriterien weiter $\mathrm{zu}$ entwickeln. Inwieweit das Menschenrechts-Monitoring der Volksanwaltschaft [2] regulär in einen qualitätssichernden Kreislauf eingebaut werden kann, ist offen. 
Tab. 1 Gemeinsame Themen von Kinder- und Jugendheilkunde, Psychiatrie und Kinder- und Jugendpsychiatrie alterangepasst und mit Vorschlägen zu sinnvollen Strukturen

\begin{tabular}{|c|c|c|c|c|c|}
\hline & PÄDIATRIE & & & & PSYCHIATRIE \\
\hline $\begin{array}{l}\text { ALTER in } \\
\text { JAHREN }\end{array}$ & Gemeinsame Themen & Gemeinsame Strukturen & $\begin{array}{l}\text { KINDER- und JUGEND- } \\
\text { PSYCHIATRIE }\end{array}$ & Gemeinsame Strukturen & Gemeinsame Themen \\
\hline $0-3$ & $\begin{array}{l}\text { Bindungsstörungen, Regula- } \\
\text { tionsstörungen, Kinderschutz }\end{array}$ & $\begin{array}{l}\text { Gegenseitiger Liäson- } \\
\text { und Konsiliardienst }\end{array}$ & - & $\begin{array}{l}\text { Präventionsambulanz; } \\
\text { gegenseitiger Liäson- } \\
\text { und Konsiliardienst }\end{array}$ & $\begin{array}{l}\text { Kinder psychisch kranker } \\
\text { Eltern }\end{array}$ \\
\hline $4-6$ & $\begin{array}{l}\text { Frühdiagnostik Autismus, } \\
\text { Ausscheidungsstörungen, } \\
\text { Psychosomatik, Somatofor- } \\
\text { me Störungen, Kinderschutz }\end{array}$ & $\begin{array}{l}\text { Gegenseitiger Liäson- } \\
\text { und Konsiliardienst }\end{array}$ & - & $\begin{array}{l}\text { Präventionsambulanz; } \\
\text { gegenseitiger Liäson- } \\
\text { und Konsiliardienst }\end{array}$ & $\begin{array}{l}\text { Kinder psychisch kranker } \\
\text { Eltern }\end{array}$ \\
\hline $6-10$ & $\begin{array}{l}\text { Schule-Leistungsstörungen, } \\
\text { Psychosomatik, Angststörun- } \\
\text { gen, ADHS, Wahrnehmungs- } \\
\text { störungen; Kinderschutz }\end{array}$ & $\begin{array}{l}\text { Gegenseitiger Liäson- } \\
\text { und Konsiliardienst; } \\
\text { gemeinsam geführte } \\
\text { Stationen }\end{array}$ & - & $\begin{array}{l}\text { Präventionsambulanz; } \\
\text { gegenseitiger Liäson- } \\
\text { und Konsiliardienst }\end{array}$ & $\begin{array}{l}\text { Kinder psychisch kranker } \\
\text { Eltern, psychisch kranke } \\
\text { Eltern }\end{array}$ \\
\hline 10-16 & $\begin{array}{l}\text { Essstörungen, Selbstverlet- } \\
\text { zendes Verhalten; Substanz- } \\
\text { missbrauch; Kinderschutz }\end{array}$ & $\begin{array}{l}\text { Gegenseitiger Liäson- } \\
\text { und Konsiliardienst; } \\
\text { gemeinsam geführte } \\
\text { Stationen }\end{array}$ & - & $\begin{array}{l}\text { Präventionsambulanz; } \\
\text { Früherkennungsam- } \\
\text { bulanz; gegenseitiger } \\
\text { Liäson- und Konsiliar- } \\
\text { dienst }\end{array}$ & - \\
\hline $16-21$ & - & - & - & $\begin{array}{l}\text { Adoleszentenstationen, } \\
\text { Transitionsambulanz; } \\
\text { gegenseitiger Liäson- } \\
\text { und Konsiliardienst }\end{array}$ & - \\
\hline
\end{tabular}

\section{Forschung}

In unserem kleinen Fach und den bisher wenigen Universitätskliniken in Österreich war die kinderund jugendpsychiatrische Forschung in Österreich nur in relativ beschränktem Ausmaß und abhängig von persönlichem Engagement möglich. Dennoch ist die Zahl und Qualität der publizierten Arbeiten bemerkenswert (siehe auch A. Karwautz in diesem Heft). Hier ist ganz klar $\mathrm{zu}$ fordern, dass die universitären Standpunkte gestärkt werden und ihnen Mittel zum Aufbau einer entsprechenden Infrastruktur für Forschung zugestanden werden. Aufgrund der kleinen Strukturen sind vernetzte Forschung, MultiCenter Studien und Einbindung in internationale Forschungsprogramme dringend nötig. Die wichtigsten Forschungsthemen sind neben der klinischen Forschung sowie dem Therapie-Outcome die Nutzung der Hirnforschungsmöglichkeiten, der Genetik und vor allem im Interesse der Kinder - die Forschung der Medikamentenwirkungen, Nebenwirkungen und der Entwicklung neuer Methoden bei der Entwicklung von Medikamenten, die möglichst nebenwirkungsfrei und gezielt erfolgen soll. Da die klassische Methodik der Medikamentenentwicklung (RCT-Modell) im Kindesalter kaum zu verantworten ist, gilt es andere Wege zu gehen, z. B. standardisierte, retrospektive und multizentrische Anwendungsbeobachtungen, wie dies im Bereich des multizentrischen therapeutischen Drugmonitorings bereits umgesetzt wird [7].

\section{Vernetzung}

Die Kinder- und Jugendpsychiatrie ist ein Fach, das aufgrund des Hineinreichens in andere Medizinische
Fächer und in verschiedene gesellschaftliche Bereiche wie den Sozialbereich, in den Familienbereich und in den Schulbereich gut definierter und klarer Kooperationsstrukturen mit den verschiedenen, sie umgebenden Institutionen bedarf.

Es sind (mindestens) drei Kooperationsfelder zu definieren:

1. Innerhalb des Fachgebietes abteilungsübergreifend, in der Ambulanz und im niedergelassenen Bereich. Vernetzung in diesem Bereich bedeutet Setting-übergreifend (Ambulanz, Tagesklink, Stationen) Behandlungskonzepte $\mathrm{zu}$ entwickeln, die durchgängig sind, von allen Bereichen in gleicher Weise qualitätsgesichert durchgeführt werden und die dem Schweregrad der Erkrankung angepasst sind, konkret heißt das z. B. ein durchgängiges Behandlungskonzept für Menschen mit Essstörungen zur Verfügung zu haben, das je nach Setting und Schweregrad angewandt wird. Dazu bedarf es auch organisatorischer und finanzieller Maßnahmen, damit die Übergänge von einem Bereich in den anderen und zurück abgestimmt und standardisiert durchgeführt werden können.

2. Mit den medizinischen Nachbarfächern Psychiatrie und Kinderheilkunde, sowie der Psychologie oder Pädagogik. Die Themen, die uns mit den beiden medizinischen Sonderfächern verbinden, sind mannigfaltig. In Tab. 1 werden einige wesentliche Themen angeführt, aufs jeweilige Alter bezogen und mit Vorschlägen für gemeinsame Strukturen ergänzt. Nur in ganz wenigen Krankenhäusern gibt es derartige Kooperationsmodelle, die zumeist nur ein oder zwei Themen umfassen. Bezüglich verbindlicher Kooperationsstandards gibt es in Österreich bisher keine Modelle. 
3. Die Kooperation mit der Kinder- und Jugendhilfe und dem gesamten Sozial- und Bildungsbereich: Trischak und CoautorInnen [26] empfehlen den Aufbau einer „standardisierten Vernetzungstätigkeit“ mit der „Etablierung von strukturierter und koordinierter, multiprofessioneller und intersektoraler Vernetzungsarbeit“. Die dafür nötige „Netzwerkarbeit“ (siehe auch die Beschreibung des NÖ Netzwerkes Industrieviertels [11, 23]) wird als eine geeignete Methode, die Zusammenarbeit effizienter und effektiver zu gestalten, gesehen [26]. Netzwerkarbeit benötigt im heutigen Gesundheitskontext aber nach wie vor die Anerkennung als solche und die entsprechende Remunerierung der Personen und die Finanzierung der nötigen Strukturen im Besonderen. Insbesondere muss die Versorgung psychisch kranker Kinder und Jugendlicher mit dem Kinder- und Jugendhilfesystem sorgfältig abgestimmt und die Verantwortlichkeiten und $\mathrm{Zu}$ ständigkeiten geklärt sein. Es darf nicht sein, dass das jeweils andere System für die Defizite des einen in die Verantwortung genommen wird, wie dies im Moment häufig der Fall zu sein scheint.

4. Kooperation mit Polizei und Justiz

Unabhängig von den spezifischen sozialen und kulturellen Kontexten ringt jede Lebensgemeinschaft mit unterschiedlichsten Methoden darum, ihre Kinder und Jugendliche angemessen zu sozialisieren, damit sie $\mathrm{zu}$ autonomen, selbstverantwortlichen Mitgliedern der Gemeinschaft werden. Daher sehen sich alle Gesellschaften mit der Herausforderung konfrontiert, angemessen und effektiv auf aggressives, impulsives und straffälliges Verhalten von Kindern und Jugendlichen $\mathrm{zu}$ reagieren. Wie schon weiter oben über die Weiterbildung für kinder- und jugendpsychiatrische Forensik beschrieben, gibt es verschiedene Ansatzpunkte der Kooperation. Es beginnt mit der häufig durch die Polizei veranlassten oder durchgeführten Einweisung in die psychiatrischen Abteilungen. Die Polizei steht hier an einer heiklen Schnittstelle, muss sie doch eine Krisensituation beurteilen, die zwar von den rechtlichen Rahmenbedingungen klar ist (siehe auch Krisenmanual Salzburg [8]), aber bezüglich ihrer inhaltlichen Qualität (psychiatrisch, sozial, pädagogisch) schwierig zu beurteilen ist. Daher bedarf es hier einer sehr guten Kooperation und Abstimmung zwischen den gesellschaftlichen Sektoren (Kinder- und Jugendhilfe, Kinder- und Jugendhilfeeinrichtungen, Polizei, Amtsärzten, Kinder- und Jugendpsychiatrie). Straffällig gewordene und inhaftierte Jugendliche stellen den zweiten, wesentlichen Berührungspunkt dar. Im Vergleich mit z. B. Schweizerischen Standards der Jugendforensik und der Behandlung straffällig gewordener Jugendliche ist Österreich bedauerlicherweise weit entfernt auch eine nur annähernd ähnliche Qualität der Betreuung ermöglichen zu können. Das Kapitel Kinderschutz ist die dritte Nahtstelle mit Polizei und Justiz. In den letzten Jahr- zehnten ist im Sinne des Opferschutzes einiges neu gestaltet worden (z. B. kontradiktorische Befragung etc.), der Umgang mit z. B. Fällen sexuellen Missbrauchs ist aber nach wie vor unbefriedigend, da nur ca. 2-3 von 10 Anzeigen zu einer Verurteilung der Täter führen. Dies liegt im Wesentlichen am Festhalten an Beweisen, die in diesen Fällen aber oft - wegen der oft jahrelangen Zwischenzeiten schwer erbringbar sind. Diese Tatsache ist für die Opfer extrem schwierig, denn sie wollen sich häufig aus diesem Grund nicht auf Anzeigen und Gerichtsverhandlungen einlassen, da überwiegend die Meinung vorherrscht, das bringe nichts. Von Seiten der Fachwelt sind große Anstrengungen unternommen worden, die Versorgung der Opfer und die Sachverständigentätigkeit in diesem Bereich zu verbessern [28]. Die innerhalb der Spitäler arbeitenden Kinderschutzgruppen sind mittlerweile hochqualifizierter Standard [25], harren aber teilweise nach wie auf finanzielle und strukturelle Unterstützung. Sie könnten und sollten - ähnlich wie in Wien - an weiteren Orten zu forensisch tätigen Ambulanzen ausgebaut werden [27].

\section{Conclusio}

In Zusammenschau all dieser Punkte entsteht durchaus eine Vision, wie wir uns die Entwicklung der Kinder- und Jugendpsychiatrie in den nächsten 20 Jahren vorstellen können. Die Versorgungsebenen werden etabliert (niedergelassen, ambulant, teilstationär, stationär) und in ausreichendem Maße und entsprechender personeller Ausstattung vorhanden sein. Zwischen Kinder- und Jugendheilkunde, Psychiatrie und Kinder- und Jugendpsychiatrie gibt es verbindliche Kooperationsabkommen mit interdisziplinär geführten Stationen und Ambulanzen. Die zahlreichen niedergelassenen Kinder- und Jugendpsychiater_innen sind in der integrierten Versorgung ihrer Patienten engagiert, sie sind Teil ihres sozial-medizinischen Gemeindeprogramms, sind vernetzt mit dem gesamten sozialen und schulischen System, betreiben Prävention, behandeln die Kinder und Jugendlichen, engagieren sich bei der Erreichung einer möglichst normalen Teilhabe ihrer Patient_innen am gesellschaftlichen Leben. Sie sind hierfür in standardisierte und mit Ressourcen und Menschen ausgestattete Netzwerke integriert. Sie sind Teil der Hilfepläneerstellung ihrer Patient_innen, organisieren Helferkonferenzen und sind „Case-Manager“. Weiters sind sie wesentlicher Bestandteil der Primärversorgungseinrichtungen, um eine möglichst frühe Diagnose und Betreuung zu ermöglichen (sekundäre und tertiäre Prävention). In allen Regionen gibt es Tageskliniken, es gibt Wohnort-nahe Reha-Plätze sowie nachgehende und auch zu Hause behandelnde multiprofessionelle Teams.

Acknowledgements Open access funding provided by Paracelsus Medical University. 
Interessenkonflikt L. Thun-Hohenstein, R. Fliedl, K. Sevecke und A. Karwautz geben an, dass kein Interessenkonflikt besteht.

Open Access Dieser Artikel wird unter der Creative Commons Namensnennung 4.0 International Lizenz (http:// creativecommons.org/licenses/by/4.0/deed.de) veröffentlicht, welche die Nutzung, Vervielfältigung, Bearbeitung, Verbreitung und Wiedergabe in jeglichem Medium und Format erlaubt, sofern Sie den/die ursprünglichen Autor(en) und die Quelle ordnungsgemäß nennen, einen Link zur Creative Commons Lizenz beifügen und angeben, ob Änderungen vorgenommen wurden.

\section{Literatur}

1. American Psychiatric Association. Diagnostisches und Statistisches Manual Psychischer Störungen -DSM-5 ${ }^{\circledR}$ : DeutscheAusgabe. Göttingen:Hogrefe; 2014.

2. Berger E. Menschenrechtsmonitoring im Kinder- und Jugendbereich. Neuropsychiatrie. 2017;31(3). doi:10.1007/ s40211-017-0244-z.

3. Boege I, Schepker R, Herpertz-Dahlmann B, Vloet TD. Hometreatment - eine effektive Alternative zu konventionellen Behandlungsformen? Z Kinder Jugendpsychiatr Psychother. 2015;43(6):411-23.

4. Bundesministerium für Gesundheit. Ärzteausbildungsordnung 2015-Fassung vom 17. April 2017.2017.

5. Cheng KF, Clark AM. Qualitative methods and patient-reported outcomes: measures development and adaptation. IntJQual Methods. 2017;16:1-3.

6. Deister A, Wilms B. Neue Behandlungsstrukturen in der Psychiatrie - Chance für eine zukunftsfähige Versorgung. Psychiatr Prax. 2015;42(8):10.

7. Egberts K, Karwautz A, Plener P, Mehler-Wex C, Kölch M, Dang SY, Taurines R, Romanos M. Pharmacovigilance in child and adolescent psychiatry. Z Kinder Jugendpsychiatr Psychother. 2015;43(1):21-8.

8. Elmer R, Thun-Hohenstein L. Krise als Chance - HandlungsleitfadenfürdieKrisenarbeitmitKindernund Jugendlichen im Bundesland Salzburg. Salzburg: Land Salzburg, Abteilung 302;2015.

9. Fegert JM. Die Kinder- und Jugendpsychiatrie und Psychotherapie als Seismograph für gesellschaftliche Entwicklungen. In: Weiß P, Peukert R, Aktion Psychisch Kranke (Hrsg.). Seelische Gesundheit und Teilhabe von Kindern und Jugendlichen braucht Hilfe. Bonn: Psychiatrie Verlag; 2011. S. 43-62.

10. Feierabend S, Plankenhorn T, Rathgeb T. JIM-Studie 2016 Jugend, Information, (Multi-)Media. 2016. https:// www.mpfs.de/studien/jim-studie/2016/. Zugegriffen: 15.04.2017.

11. Fliedl R. Krisenmanual für Kinder und Jugendliche im Industrieviertel. Hinterbrühl: Karl Landsteiner Gesellschaft; 2017.

12. Fliedl R, Sevecke K. Zur Psychodynamik von Organisation und Kooperation in der Kinder- und Jugendpsychiatrie. Neuropsychiatrie. 2017;31(3). doi:10.1007/s40211017-0247-9.

13. Fülöp G, et al. Österreichischer Strukturplan Gesundheit 2012. Wien: Bundesministerium für Gesundheit; 2012.

14. General Assembly of United Nations. UN-Kinderrechtskonvention-Übereinkommen über die Rechte des Kindes. 1989.
15. General Assembly of United Nations. Convention on the rights of persons with disabilities (CRPD). 2006.

16. Grüner P. Die McDonaldisierung der Medizin. Forum Med. 2017;3:7-8.

17. Hartl C, Karwautz A. Zehn Jahre Kinder- und Jugendpsychiatrie in Österreich: ein neues ärztliches Sonderfach in den Strukturen des Gesundheitswesens. Neuropsychiatrie. 2017;31(3). doi:10.1007/s40211-017-0235-0

18. KarwautzA,Purtscher-PenzAK,HochgattererP, Kienbacher C. Child and adolescent psychiatry in Austria. Eur Child Adolesc Psychiatry. 2015;24:359-60.

19. Kräuter G. Eklatante Versorgungsdefizitein derWiener Kinder-undJugendpsychiatrie.2017.http://volksanwaltschaft. gv.at/artikel/versorgungsmangel-in-der-kinder-und-ju gendpsychiatrieVolksanwaltschaft.Zugegriffen:15.04.2017.

20. Maio G. Medizin in einer Gesellschaft, die kein Schicksal duldet. eine Kritik des Machbarkeitsdenken der modernen Medizin.ZMed Ethik. 2011;57:79-98.

21. Pickel G. Bedrohungsgefühle versus vertrauensbildende Kontakte: Religiöser Pluralismus, religiöses Sozialkapital und soziokulturelle Integration. In: Pollack D, Tucci I, ZiebertzHG (Hrsg.). Religiöser Pluralismus im Fokus quantitativer Religionsforschung. Wiesbaden:VS; 2012. S. 221-61.

22. Shannon S. The premise, practice, and promise of integrative child and adolescent psychiatry. Psychiatr Times. 2016;33:1-3.

23. Tatzer E, Damm L, Winter B, Fliedl R. Interdisziplinäre Zusammenarbeit bei der Behandlung und Betreuung von Kindern und Jugendlichen mit komplexem Hilfebedarf Das Kindernetzwerk Industrieviertel in Niederösterreich. Resonanzen. 2013;1(2):138-51.

24. Thun-Hohenstein L. Partizipation von Kindern und Jugendlichen an einer Kinder+Jugendpsychiatrie. Pädiatr Pädol. 2014;49(Suppl 1):42-7.

25. Thun-Hohenstein L. Kinderschutzgruppen. In:Völkl-Kernstock S, Kienbacher C (Hrsg.). Forensische Arbeit mit Kindern und Jugendlichen. Vienna: Springer;2017. S. 115-23.

26. Trischak C, Bachler S, Ruda R, Hochwallner C. Nahtstellenmanagement in der Versorgung von Kindern und Jugendlichen mit psychischen Auffälligkeiten. Wien: Competence Center Integrierte Versorgung Wiener Gebieteskrankenkasse; 2016. http:/ / www.cciv.at/cdscontent/load? contentid $=10008.637783 \&$ version $=1487064754$.

27. Völkl-Kernstock S, Grundner A, Klinger D. Forensik und Traumadiagnostik Ambulanz. 2017. https://kjp. meduniwien.ac.at/patientinneninformationen/spezialam bulanzen/forensik-und-traumadiagnostik-ambulanz/. Zugegriffen:30.06.2017.

28. Völkl-Kernstock S, Kienbacher C. Forensische Arbeit mit Kindern und Jugendlichen: Praxishandbuch für die interdisziplinäreZusammenarbeit. Vienna: Springer; 2017.

29. Wagner G, Zeiler M, Waldherr K, Philipp J, Truttmann S, TreasureJL, KarwautzA. Mentalhealth problemsinAustrian adolescents: a nationwide, two-step epidemiological study applying DSM-V criteria. Eur Child Adolesc Psychiatry. 2017; doi:10.1007/s00787-017-0999-6.

30. Wolf K, Kossakck P. Qualitätsmanagement im Krankenhaus verstehen und anwenden. Die ISO 9001:2015 in Kliniken und anderen Gesundheitseinrichtungen des Gesundheitswesens. Düsseldorf: Symposium Publishing; 2016.

31. Zhao J, Gao S, Wang J, Liu X, Hao Y. Differentiation between two health car concepts: person-centered and patientcentered care. Int J Nurs Sci. 2016;3:398-402. 